\title{
ANALYSIS OF ELLIS FLUID IN WIRE COATING
}

\author{
MUHAMMAD AYAZ, TAZA GUL, SAEED ISLAM, MUHAMMAD ALTAF KHAN \\ Department of Mathematics, Abdul Wali Khan, University Mardan, Khyber Pakhtunkhwa, Pakistan
}

Revised May 2015

ABSTRACT. The focus of the present work is concerned with the study of wire coating analysis using Ellis fluid. Using the binomial series method, closed form solution is obtained and further in the closed form of solution, we discussed a special case for the parameter, and obtained the expressions for axial velocity, shear stress, average velocitf, volume flow rate and temperature distribution. Thickness of the coated wire and force on the total wire are also calculated. The effect of different parameters of interest on the variation of the velocity profile, volume flow rate, shear stress, radius of coated wire and force on the total wire are discussed and presented graphically.

Keywords: Wire Coating, Ellis Fluid, Pressure Type Die, Binomial Series Method.

1. Introduction. Wire coating is used for the purpose of high and low voltage, for the protection of humans, for the processing of signals such as cable and telephone wires and for the protection against corrosion. It is performed due the drag of wire in the coating unit filled with molten polymers, and hence wire is coated due to the shear stress between the wire and molten polymer. Wire coating is one of the important chemical process in which different types of polymers are used. The coating of wire depends on geometry of die, viscosity of fluid, temperature of wire and molten polymer used for coating. Wire coating have many applications in the field of chemical and industrial engineering. Many authors have studied wire coating phenomena.

The basic concept of modeling the wire coating for viscous fluid is given by Denn and Middleman [1, 2]. Akhter and Hashmi $[3,4]$ have studied wire coating using power law fluid and have investigated the effect of change in viscosity. Fenner and Williams [5] carried out an analysis of the flow in the tampering section of a pressure type die. Siddiqui et al [6] studied wire coating extrusion in a pressure type die in the flow of third grad fluid. They obtained the numerical solutions for the pressure and velocity profiles in the die. Sajjid et al [7] studied the wire coating with oldroyd 8-constant fluid and give the solution for velocity field in the form of series. Gagley and Story [8] provided numerical simulations for Newtonian fluid in the form of dimensionless parameters characterizing the wire speed, die dimension, radial position, shear rate and melt viscosity. The analysis of drag flow of coated polymer outside pressure type die was carried out by Kasajima and Katsuhiko Ito [9]. They derived the expressions for velocity and temperature field. Moreover, they found average velocity, volume flow rate and discussed some cases for constant temperature on the boundaries. Han and Rao [10] discussed the rheology of wire coating extrusion. Casewell and Tanner [11] made first attempt to analyze the isothermal flows of Newtonian and power law fluids inside the wire coating dies, without making use of lubrication approximation theory (LAT). Roy and Dutt [12] further investigated and suggested the theory for wire coating by withdrawal from a power law fluid and tested with experimental data on Newtonian and pseudoplastic fluids. Evan Mitsoulis [13] studied fluid flow and heat transfer in wire coating. Recently, Rehan et al [14] made the first attempt to analyze the isothermal flows of unsteady second grad fluid inside the wire coating die with oscilating boundary condition using the Optimal Homotopy Asymptotic Method. Rehan et al [15] studied the wire coating analysis with oldroyd 8-constant fluid by Optimal Homotopy Asymptotic Method, and obtained the solution for velocity field. Rehan et al [16] also studied the exact solution of differential equation arising in the wire coating analysis of an unsteady second grad fluid. Mckelvey [17] and Paton et al [18] have analyzed the flow of Newtonian and power law fluid models in the 
wire coating analysis, and obtained the expressions for the flow rate, shear rate and velocity distribution along the radial direction.

According to the best of our knowledge the mathematical modeling using an Ellis fluid model of wire coating process with no-slip boundary conditions has not been studied. As the Ellis fluid has many industrial applications like waterborne coating and metallic automotive base coating where polymeric suspensions are used, lubricating greases, psueodoplastic fluids and drilling fluids are its industrial applications [20, 21]. Thus we modeled the wire coating process by considering Ellis fluid as melt and develop the resulting differential equation by using Binomial series technique. The non-linear differential equations governing the model are made dimensionless and solved for velocity and temperature distribution. We derive closed form of solution in dimensionless form for velocity field, shear stress, volume flow rate, thickness of coated wire, force on total wire and temperature distribution.

2. Basic Governing Equations. The basic laws of conservation of mass, conservation of momentum governing the flow of incompressible Ellis fluid model with thermal effect is

$$
\begin{aligned}
& \nabla . V=0, \\
& \rho \frac{\mathrm{D} V}{\mathrm{Dt}}=-\nabla P+\operatorname{div} S+\rho f, \\
& \rho \mathrm{C}_{\mathrm{p}} \frac{\mathrm{D} \theta}{\mathrm{Dt}}=\mathrm{k} \nabla^{2} \theta+S . \nabla V,
\end{aligned}
$$

where $\rho$ is constant density, $V$ is velocity vector, $f$ is body force, $p$ is pressure, $S$ is the extra stress tensor and $\frac{\mathrm{D}}{\mathrm{Dt}}$ is the material derivative. As discussed in [19] the stress tensor defining the Ellis fluid is given by

$$
S=\eta A_{1},
$$

Where

$$
\frac{1}{\eta}=\frac{1}{\eta_{0}}\left[1+\left|\frac{\sqrt{\frac{1}{2}(S S S)}}{\frac{S_{1}}{2}}\right|^{\alpha-1}\right],
$$

And

$$
A_{1}=\nabla V+(\nabla V)^{T}
$$

Where $\eta$ is the apparent viscosity, $\eta_{0}$ is zero shear viscosity, $S_{\frac{1}{2}}$ represents the value of shear stress at which the apparent viscosity has dropped to half its zero shear value, and lastly the index $\alpha$ is a measure of the degree of shear thinning behavior that is the greater the value of $\alpha$ greater is the extent of shear thinning.

3. Formulation and solution of the problem. Consider steady, parallel, laminar flow of an incompressible Ellis fluid flowing in stationary cylindrical die of length $\mathrm{L}$ and of radius $R_{\mathbb{d}}$. The wire of radius $R_{w}$ is translating along the center line of the die with uniform velocity $U_{w}$ as shown in the Fig. 1 below. The wire and die are taken to be concentric. The fluid is acted upon by a constant pressure gradient $\frac{d p}{d z}$ in the z-direction. The drag of wire and pressure gradient along the axial direction is responsible for the flow of fluid. We choose the cylindrical coordinate system $(r, \theta, z)$ such that $\mathrm{z}$-axis is along the centre of the die and r-axis is normal to z-axis. 


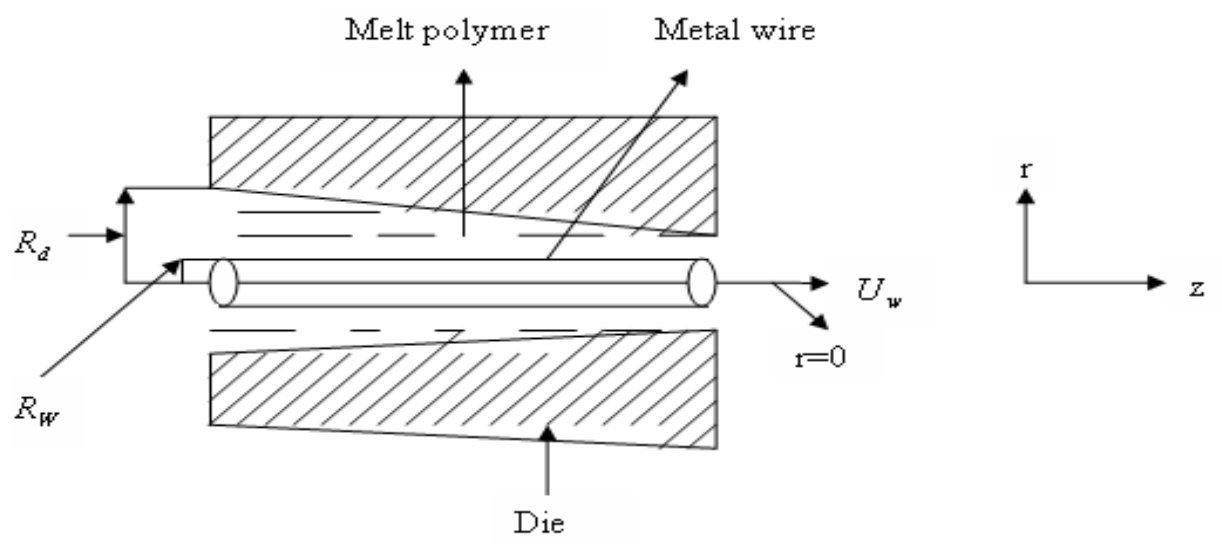

Fig. 1 : Schematic profile of wire coating in pressure type die.

The boundary conditions for the problem on the velocity field and temperature distribution are given by

$$
\begin{array}{ll}
\mathrm{w}\left(R_{w}\right)=\mathrm{U}_{\mathrm{w}}, & \mathrm{w}\left(R_{\mathbb{d}}\right)=0, \\
\Theta\left(R_{w}\right)=\theta_{\mathrm{w}}, & \Theta\left(R_{\mathbb{d}}\right)=\theta_{\mathrm{d}},
\end{array}
$$

The fluid velocity field and temperature distributions are considered as

$$
V=[0,0, w(r)], \quad S=\mathrm{S}(\mathrm{r}), \quad \Theta=\Theta(\mathrm{r}),
$$

Using the assumptions continuity Eqn. 1 satisfied identically, the momentum Eqn. 2 and energy Eqn. 3 in the absence of body forces reduces to

$$
\begin{gathered}
\frac{\partial P}{\partial y}=0, \\
\frac{\partial P}{\partial \theta}=0, \\
\frac{\partial p}{\partial z}=\frac{1}{\frac{d}{d r}}\left(r S_{r z}\right), \\
\mathrm{k}\left(\frac{d^{2}}{d r^{2}}+\frac{1}{y} \frac{d}{d r}\right) \theta+S_{r z} \frac{d w}{d r}=0,
\end{gathered}
$$

From Eqns. 10-12 it is concluded that ' $p$ ' is function of ' $z$ ' only, also assume that there is no variation in pressure gradient along the axial direction. Thus we have

$$
\frac{\partial p}{\partial z}=B, \text { where }
$$

$\mathrm{B}$ is a constant.

Thus Eqn. 12 reduces to the form

$$
\text { Integrating w.r.t ' } r \text { ' we get } \quad S_{r z}=\left(\frac{B}{2} r+\frac{k_{1}}{r}\right),
$$

Where $\mathrm{k}_{1}$ is an arbitrary constant of integration.

Now by the use of Eqn. 9, in the constitutive equations, the combination of Eqn. 4 and Eqn. 5 is given by

$$
\frac{d w}{d y}=\frac{1}{\eta_{0}}\left[1+\left|\frac{S_{y z}}{\frac{S_{1}}{2}}\right|^{\alpha-1}\right] \cdot S_{y z},
$$

Substituting Eqn. 14 in Eqn. 15 and for relaxing the absolute condition, we have 


$$
\frac{d w}{d y}=\frac{1}{\eta_{0}}\left(\frac{\mathrm{B}}{2} \mathrm{r}+\frac{\mathrm{k}_{1}}{r}\right)+\frac{\mathrm{k}_{1}{ }^{\alpha}}{\eta_{0} \mathrm{r}^{\alpha} \frac{S_{1}^{\alpha-1}}{2}}\left(1+\frac{\mathrm{B}}{2 \mathrm{k}_{1}} r^{2}\right)^{\alpha}
$$

Using the Binomial series formula and taking $\left|\frac{B r^{2}}{2 \mathrm{k}_{1}}\right|<1$, we obtain

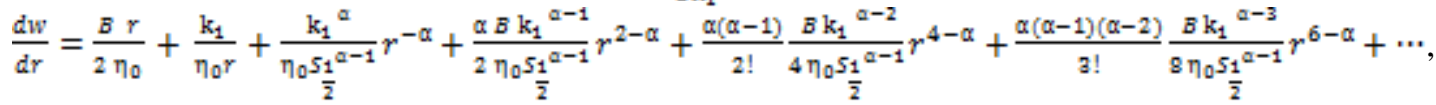

Now introducing the following dimensionless parameters in Eqn. 17

$$
\begin{aligned}
& w^{*}=\frac{\mathrm{w}}{\mathrm{U}_{\mathrm{W}}}, r^{*}=\frac{\mathrm{r}}{\mathrm{R}_{\mathrm{W}}}, \hat{\mathrm{K}}_{1}=\frac{2 \mathrm{k}_{1}}{B \mathrm{R}_{\mathrm{W}}{ }^{2}}, D e_{c}=\frac{\lambda \mathrm{U}_{c}}{\mathrm{R}_{\mathrm{W}}}, X=\frac{\mathrm{U}_{c}}{\mathrm{U}_{\mathrm{W}}}, \frac{\mathrm{R}_{\mathrm{d}}}{\mathrm{R}_{\mathrm{W}}}=\delta>1, \\
& \lambda=\frac{1}{\frac{S_{1}{ }^{\alpha-1}}{2}}, B r=\frac{\eta_{0} \mathrm{U}_{W}{ }^{2}}{k\left(\theta_{d}-\theta_{w}\right)}, \mathrm{U}_{\mathrm{c}}=\frac{-B \mathrm{R}_{\mathrm{W}}{ }^{2}}{\mathrm{~g} \eta_{0}},
\end{aligned}
$$

Where $D e_{c}$ is Deborah number based on velocity scale $U_{c}, X=\frac{U_{c}}{\mathbb{V}_{w}}$ has physical meaning of nondimensional pressure gradient and $B r$ is the Brinkman number. From these dimensionless parameters

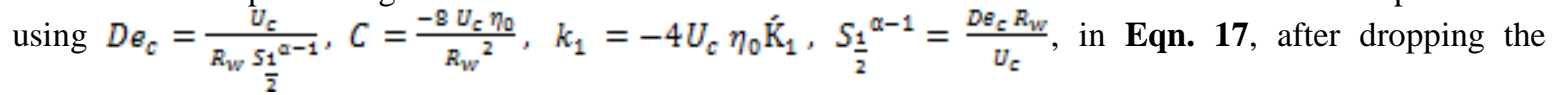
asterisks we obtain

$\frac{d w}{d y}=\frac{-4 \mathrm{U}_{c}}{\mathrm{R}_{\mathrm{W}}{ }^{2}} r-\frac{4 \mathrm{U}_{c} \hat{\mathrm{K}}_{1}}{\gamma}+(-4)^{\alpha} D e_{c} \mathrm{R}_{\mathrm{W}} \eta_{0}{ }^{\alpha-1} \mathrm{U}_{\mathrm{c}}{ }^{\alpha-1} \hat{\mathrm{K}}_{1}^{\alpha} \mathrm{r}^{-\alpha}+\alpha(-4)^{\alpha} \frac{D \theta_{c}}{\mathrm{R}_{\mathrm{W}}} \eta_{0}{ }^{\alpha-1} \mathrm{U}_{\mathrm{c}}{ }^{\alpha-1} \hat{\mathrm{K}}_{1}^{\alpha-1} \mathrm{r}^{2-\alpha}+$

$\frac{\alpha(\alpha-1)}{2 !}(-4)^{\alpha} \frac{D g_{c}}{\mathrm{R}_{\mathrm{w}}{ }^{3}} \eta_{0}{ }^{\alpha-1} \mathrm{U}_{\mathrm{c}}{ }^{\alpha-1} \hat{\mathrm{K}}_{1}^{\alpha-2} \mathrm{r}^{4-\alpha}+\frac{\alpha(\alpha-1)(\alpha-2)}{\mathrm{a} !}(-4)^{\alpha} \frac{D e_{c}}{\mathrm{R}_{\mathrm{w}}{ }^{5}} \eta_{0}{ }^{\alpha-1} \mathrm{U}_{\mathrm{c}}{ }^{\alpha-1} \hat{\mathrm{K}}_{1}{ }^{\alpha-9]} \mathrm{r}^{6-\alpha}+\cdots$

Taking $\mathrm{R}_{\mathrm{w}}=1, \mathrm{U}_{\mathrm{w}}=1$, then $\mathrm{U}_{\mathrm{c}}=\mathrm{X}_{,} \mathrm{R}_{\mathrm{d}}=\delta$, Eqn. 18 yields

$\frac{d w}{d r}=-4 X r-4 X \hat{\mathrm{K}}_{1} \frac{1}{r}+(-4)^{\alpha} D e_{c} \eta_{0}{ }^{\alpha-1} \mathrm{X}^{\alpha-1} \sum_{k=0}^{\mathrm{s}=0}\left(\begin{array}{l}\alpha \\ k\end{array}\right) \hat{\mathrm{K}}_{1}^{(\alpha-k)} r^{(2 k-\alpha)}$,

Eqn.19 represent the axial velocity gradient in non-dimensional form and hence the boundary conditions given in Eqn. 7, reduces to

Now

$$
w(1)=1, w(\delta)=0,
$$

The temperature distribution from Eqn. 13 in non-dimensional form can be obtain as

$$
\begin{gathered}
\mathrm{k}\left(\frac{d^{2}}{d r^{2}}+\frac{1}{r} \frac{d}{d r}\right) \theta+S_{r z} \frac{d w}{d r}=0, \\
\text { using } S_{r z}=\left(\frac{\mathrm{B}}{2} \mathrm{r}+\frac{\mathrm{k}_{1}}{r}\right) \text {, we obtain } \\
\mathrm{k}\left(\frac{d^{2}}{d r^{2}}+\frac{1}{r} \frac{d}{d r}\right) \theta+\left(\frac{\mathrm{B}}{2} \mathrm{r}+\frac{\mathrm{k}_{1}}{r}\right) \frac{d w}{d r}=0, \\
\frac{k}{r} \frac{d}{d r}\left(r \frac{d \theta}{d r}\right)+\frac{1}{r}\left(\frac{\mathrm{B}}{2} \mathrm{r}^{2}+\mathrm{k}_{1}\right) \frac{d w}{d r}=0, \\
k \frac{d}{d r}\left(r \frac{d \theta}{d r}\right)+\left(\frac{\mathrm{B}}{2} \mathrm{r}^{2}+\mathrm{k}_{1}\right) \frac{d w}{d r}=0,
\end{gathered}
$$

Using $B=\frac{-8 \mathrm{U}_{\mathrm{c}} \eta_{0}}{\mathrm{R}_{\mathrm{w}}{ }^{2}}, \mathrm{k}_{1}=-4 \mathrm{U}_{\mathrm{c}} \eta_{0} \hat{\mathrm{K}}_{1}, \mathrm{R}_{\mathrm{w}}=1, \mathrm{U}_{\mathrm{w}}=1, \mathrm{U}_{\mathrm{c}}=\mathrm{X}, \frac{\eta_{\mathrm{o}}}{k}=B r$, in last equation, we obtain

$$
\frac{d}{d y}\left(r \frac{d \theta}{d y}\right)-4 B r X\left(r^{2}+\tilde{K}_{1}\right) \frac{d w}{d y}=0 \text {, }
$$

With the boundary conditions given in Eqn. 8, reduces to $\theta(1)=0, \theta(\delta)=1$,

Here we list the basic formulas related to the wire coating analysis for future use in our work.

The average velocity of fluid inside the die during coating process is given by

$$
w_{\text {awe }}=\frac{2}{\left(R_{d}^{2}-R_{w}^{2}\right)} \int_{R_{w}}^{K_{d}} r w(r) d r
$$

At some control surface downstream, the volume flow rate of coating is given by

$$
\mathrm{Q}=\pi \mathrm{U}_{\mathrm{w}}\left(\mathrm{R}_{\mathrm{c}}{ }^{2}-\mathrm{R}_{\mathrm{w}}{ }^{2}\right)
$$

Where $R_{c}$ is the radius of the coated wire.

And to find the Volume flux, we use the formula given by the equation

$$
\mathrm{Q}=\int_{\mathrm{R}_{\mathrm{w}}}^{\mathrm{K} d} 2 \mathrm{mr} \mathrm{w}(\mathrm{r}) d r,
$$

According, from both the Eqns. 24-25 the thickness of the coated wire can be obtained as

$$
\mathrm{R}_{\mathrm{c}}=\left(\mathrm{R}_{\mathrm{w}}^{2}+\frac{2}{\mathrm{U}_{\mathrm{w}}} \int_{\mathrm{R}_{\mathrm{w}}}^{\mathrm{R}_{\mathrm{d}}} \mathrm{rw}(\mathrm{r}) \mathrm{dr}\right)^{\frac{1}{2}}
$$


The force on the wire is computed by determining the shear stress at the wire surface, which is given by

$$
\left.S_{y z}\right|_{y=\mathrm{R}_{w}}=\left.\left(\frac{\mathrm{B}}{2} \mathrm{r}+\frac{\mathrm{k}_{1}}{r}\right)\right|_{r=\mathrm{R}_{w}},
$$

The force exerted by the fluid on the total wire surface is given by the formula

$$
F_{w}=\left.2 \pi \mathrm{R}_{\mathrm{w}} \mathrm{L} \cdot S_{r z}\right|_{y=\mathrm{R}_{\mathrm{w}}},
$$

Using $R_{w}=1, U_{w}=1$, then $U_{c}=X, R_{d}=\delta$, the system of Eqns. 23-28 reduces to

$$
\begin{aligned}
& \mathrm{w}_{\mathrm{aWe}}=\frac{\mathrm{w}_{\mathrm{avv}}\left(\mathrm{R}_{\mathrm{d}}{ }^{2}-\mathrm{R}_{\mathrm{w}}{ }^{2}\right)}{2 \mathrm{R}_{\mathrm{w}}{ }^{2} \mathrm{U}_{\mathrm{w}}}, \\
& \mathrm{w}_{\text {ave }}=\int_{1}^{\delta} \mathrm{rw}(\mathrm{r}) \mathrm{dr} \text {, } \\
& Q^{*}=\frac{Q}{2 \pi R_{W}{ }^{2} U_{w}},
\end{aligned}
$$

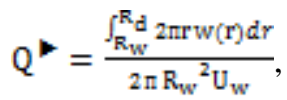

$$
\begin{aligned}
& \mathrm{Q}^{*}=\int_{1}^{8} \mathrm{rw}(\mathrm{r}) \mathrm{dr}, \\
& \mathrm{R}_{\mathrm{c}}=\frac{\mathrm{R}_{\mathrm{c}}}{\mathrm{R}_{\mathrm{w}}}, \\
& \mathrm{R}_{\mathrm{c}}=\left(1+2 \mathrm{Q}^{\prime}\right)^{\frac{1}{2}}, \\
& \left.S_{r z}\right|_{r=1}=\left.\frac{R_{W} s_{r z}}{\eta_{0} U_{c}}\right|_{r=1}, \\
& \left.S_{r z}\right|_{r=1}=-4\left(1+\check{K}_{1}\right) \text {, } \\
& \left.F_{W V}\right|_{Y=1}=\left.\frac{F_{V V}}{2 \mathrm{~m} \eta_{0} \mathrm{~L} \mathrm{U}_{c}}\right|_{Y=1}, \\
& \left.F_{W V}\right|_{r=1}=-4\left(1+\hat{K}_{1}\right) \text {, } \\
& \left.F_{W V}\right|_{r=1}=\left.s_{r z}\right|_{r=1},
\end{aligned}
$$

The stress component $S_{y z}$ in dimensionless form is given by

$$
\begin{aligned}
S_{r z} & =\frac{\mathbb{R}_{\mathrm{w}} s_{r z}}{\eta_{0} \mathrm{U}_{c}}, \\
S_{y z} & =\frac{1}{\eta_{0} \mathrm{U}_{c}}\left(\frac{\mathrm{B}}{2} \mathrm{r}+\frac{\mathrm{k}_{1}}{r}\right), \\
S_{y z} & =-4\left(r+\frac{\mathbb{K}_{1}}{r}\right)
\end{aligned}
$$

Now

Integrating Eqn. 19 with respect to r, we obtain

$w(r)=-2 X r^{2}-4 X \dot{K}_{1} \ln (r)+(-4)^{\alpha} D e_{c} \eta_{0}{ }^{\alpha-1} X^{\alpha-1} \sum_{k=0}^{\infty e}\left(\begin{array}{l}\alpha \\ k\end{array}\right) \hat{K}_{1}^{(\alpha-k)} \frac{r^{(2 k-\alpha+1)}}{(2 k-\alpha+1)}+k_{2}$,

Where $k_{2}$ is another constant of integration to be determined.

The expression of velocity profile given in Eqn. 35 involves two constants $\tilde{K}_{1}$ and $k_{2}$ that can be determined explicitly by using the boundary conditions, $\mathrm{w}(1)=1, \mathrm{w}(\delta)=0$, also this equation contains an infinite series for which we want to discuss a special case by taking $\alpha=0$ in order to find the constant $\overleftarrow{\mathrm{K}}_{1}$ and $k_{2}$.

Special Case: when $\alpha=2 x$ in Eqn. 35, we obtain 
Inserting the boundary conditions from Eqn. 20 into Eqn. 35, we end up with a quadratic equation,

$$
\hat{\mathrm{K}}_{1}^{2}+Q_{0} \hat{\mathrm{K}}_{1}+Q_{1}=0
$$

with coefficients,

$$
\begin{aligned}
Q_{0}=\frac{s_{1}}{s_{2}}, \quad Q_{1} & =\frac{s_{0}}{s_{2}^{x}} \\
S_{0} & =\frac{16}{a} D e_{c} \eta_{0} X\left(1-\delta^{a}\right)+2 X\left(\delta^{2}-1\right)-1_{x} \\
S_{1} & =4 X\left(\ln (\delta)+8 D e_{c} \eta_{0}(1-\delta)\right) . \\
S_{2} & =16 D e_{c} \eta_{0} X\left(\frac{1}{6}-1\right) .
\end{aligned}
$$

The real root to the quadratic Eqn. 36 can be obtain by taking the discriminant $Q_{0}{ }^{2}-4 Q_{1}=0$.

we get,

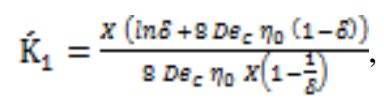

The second constant of integration $k_{2}$ appeared in the axial velocity profile can be determined by the following method.

If both boundaries are stationary or moving then use of any boundary condition gives the second constant $k_{2}$ on the other hand if one boundary is stationary and other is moving with constant speed, then $k_{2}$ can be determined from the condition on the stationary boundary, otherwise the solution will not satisfy both the boundary conditions. Thus we determine the second constant $k_{2}$ from Eqn. 35 by setting the no-slip condition at the die wall as,

$$
k_{2}=2 \delta^{2} X+4 \hat{K}_{1} X \ln (\delta)-\frac{16}{a} D e_{c} \eta_{0} X \delta^{a}-32 D e_{c} \eta_{0} X \hat{K}_{1} \delta+\frac{16 D e_{c} \eta_{0} X \hat{K}_{1}^{2}}{g},
$$

\section{The velocity profile in dimensionless form}

$$
w(r)=-2 X r^{2}-4 X \hat{\mathrm{K}}_{1} \ln (r)+16 D e_{c} \eta_{0} X\left(\frac{r^{3}}{a}+2 \hat{\mathrm{K}}_{1} r-\frac{\hat{\mathrm{K}}_{1}{ }^{2}}{r}\right)+k_{2}
$$

Volume flow rate $Q^{*}=\int_{1}^{8} \mathrm{r} w(\mathrm{r}) \mathrm{dr}$ in dimensionless form is

$Q^{2}=-\frac{x}{2}\left(\delta^{4}-1\right)-X \hat{K}_{1}\left(2 \delta^{2} \ln (\delta)-\delta^{2}+1\right)+16 D e_{c} \eta_{0} X\left(\frac{1}{15}\left(\delta^{5}-1\right)+\frac{2}{\mathrm{a}} \hat{\mathrm{K}}_{1}\left(\delta^{\mathrm{a}}-1\right)-\hat{\mathrm{K}}_{1}{ }^{2}(\delta-\right.$

1)) $+\frac{k_{2}}{2}\left(\delta^{2}-1\right)$

Thickness of the coated wire $R_{c}=\left(1+2 Q^{\vee}\right)^{\frac{1}{2}}$ in dimensionless form is

$\mathrm{R}_{\mathrm{c}}=\left(1-X\left(\delta^{4}-1\right)-2 X \hat{K}_{1}\left(2 \delta^{2} \ln (\delta)-\delta^{2}+1\right)+32 D e_{0} \eta_{0} X\left(\frac{1}{15}\left(\delta^{5}-1\right)+\frac{2}{\mathrm{a}} \hat{\mathrm{K}}_{1}\left(\delta^{\mathrm{a}}-1\right)-\right.\right.$

$\left.\left.\tilde{\mathrm{K}}_{1}^{2}(\delta-1)\right)+k_{2}\left(\delta^{2}-1\right)\right)^{\frac{1}{2}}$

\section{The force on the total wire in dimensionless form is}

$$
F_{w V}=-4\left(1+\hat{K}_{1}\right),
$$

This expression shows an important result that the force on the wire is constant throughout the domain and which depends upon the parameters involved in constant $\stackrel{K}{1}_{1}$.

To find Temperature distribution we use the value of $\frac{d w}{d y}$ from Eqn. 19 in Eqn. 21 with boundary conditions, 


$$
\begin{aligned}
& \theta(1)=0, \theta(\delta)=1 \text {, we obtain } \\
& \frac{d}{d r}\left(r \frac{d \theta}{d r}\right)-4 B r X\left(\mathrm{r}^{2}+\hat{\mathrm{K}}_{1}\right)\left(-4 r X-4 \hat{\mathrm{K}}_{1} X \frac{1}{r}+16 D e_{c} \eta_{0} X\left(r^{2}+2 \tilde{\mathrm{K}}_{1}-\hat{\mathrm{K}}_{1}{ }^{2} \frac{1}{r^{2}}\right)\right)=0 \text {, } \\
& \theta(\mathrm{r})=-4 B r X\left(\frac{x}{4}\left(r^{4}-1\right)+2 \hat{\mathrm{K}}_{1} X\left(r^{2}-1\right)+2 \hat{\mathrm{K}}_{1}^{2} X \ln (r)^{2}-16 D e_{c} \eta_{0} X\left(3 \hat{\mathrm{K}}_{1}^{2}(r-1)+\right.\right. \\
& \left.\left.\hat{\mathrm{K}}_{1}^{a}\left(\frac{1}{r}-1\right)+\frac{1}{25}\left(r^{5}-1\right)+\frac{\hat{k}_{1}}{a}\left(r^{a}-1\right)\right)\right)+4 B r X \frac{\ln (r)}{\ln (d)}\left(\frac{x}{4}\left(\delta^{4}-1\right)+2 \hat{\mathrm{K}}_{1} X\left(\delta^{2}+\ln (\delta)-1\right)-\right. \\
& \left.16 D e_{c} \eta_{0} X\left(3 \hat{\mathrm{K}}_{1}^{2}(\delta-1)+\frac{\hat{\mathrm{k}}_{1}^{3}}{9 g}\left(\delta^{4}-9 \delta+9\right)+\frac{1}{25}\left(\delta^{5}-1\right)+\frac{\hat{\mathrm{k}}_{1}}{9}\left(2 \delta^{2}-3\right)\right)\right)+\frac{\ln (r)}{\ln (\delta)}
\end{aligned}
$$

4. The results and discussion. Eqn. 19 and Eqn. 21 along with the boundary conditions given in Eqn. 20 and Eqn. 22 were solved for velocity field and temperature distribution by considering a special case for the parameter $\alpha$. Moreover, the volume flow rate, average velocity, thickness of the coated wire, shear stress and force on total wire are derived using velocity field. All the results are observed physically through Figs. (2)-(10). The effect of dimensionless parameters $D e_{e^{x}} X$ and Brinkman number $B r$ are discussed. Figs. (2)-(3), represent the velocity profiles as a function of ' $r$ ' for several values of dimensionless numbers $X$ and $D e_{c}$. In Figs. (2) we varied the ratio between the pressure drop and speed of wire $\left(X=\frac{\mathbb{U}_{c}}{\mathbb{v}_{w}}\right)$, that is, $X=1.1,1.3,1.7,2.2$, fixing $\delta=2, D e_{c}=0.01, \eta_{0}=0.7$, which shows that the rise in pressure gradient increases the speed of flow. Fig. (3) is sketched for $D e_{c}=3,5,8,12$ fixing $\delta=2, \eta_{0}=0.7, X=0.5$. It is obvious from the Figs. (2)-(3) that velocity decreases with increase in dimensionless numbers $X$ and decreases for $D e_{c}$. Fig. (4) is plotted for variation of shear stress for different values of $D e_{c}$. It is to be noted that an increase in the parameter $D e_{c}$, shear stress increases which is insignificant for small and large values of $D e_{c}$. The Fig. (5) is plotted for variation of volume flow rate for different values of $D e_{c}$ fixing $X=0.5, \eta_{0}=0.7$. Here it is observed that the flow rate decreases for increasing values of $D e_{c}$. The thickness of the coated wire is plotted in the Fig. (6) for different values of $D e_{c}$ fixing $X=0.5, \eta_{0}=0.7$, that also show decrease in the thickness. The dimensionless force on the total wire versus $\delta$ is sketched in the Fig. (7) for various values of $D e_{c}$ fixing $X=0.5, \eta_{0}=0.7$. It is to be noted that the graph show increase in the force on total wire as $\delta$ increases. In Figs. (8)-(10), we plotted the dimensionless temperature profile $\theta(\mathrm{r})$ versus $\delta$ with selected sets of parameters $B r_{s} D e_{e^{v}}$ and $X$. It is obvious from the Figs. (8)-(10) that temperature increases as these parameters increases, It is also observed that temperature increases very rapidly for the increasing values of the parameters $X$ and $D e_{e}$. Thus we concluded that the temperature distribution increases as the dimensionless numbers $B r_{s} D e_{c}$ and $X$ increases. 


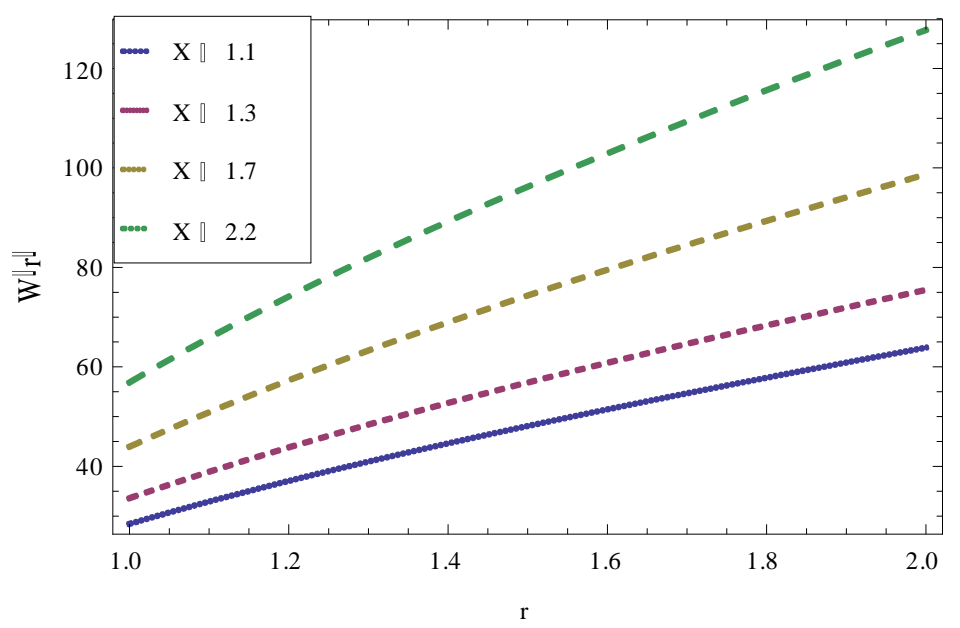

Figure 2: Dimensionless velocity profiles for different values of $X=1.1,1.3,1.7,2.5,2.2$ at fixed values of $\delta=2, D e_{c}=0.01, \eta_{0}=0.7$.

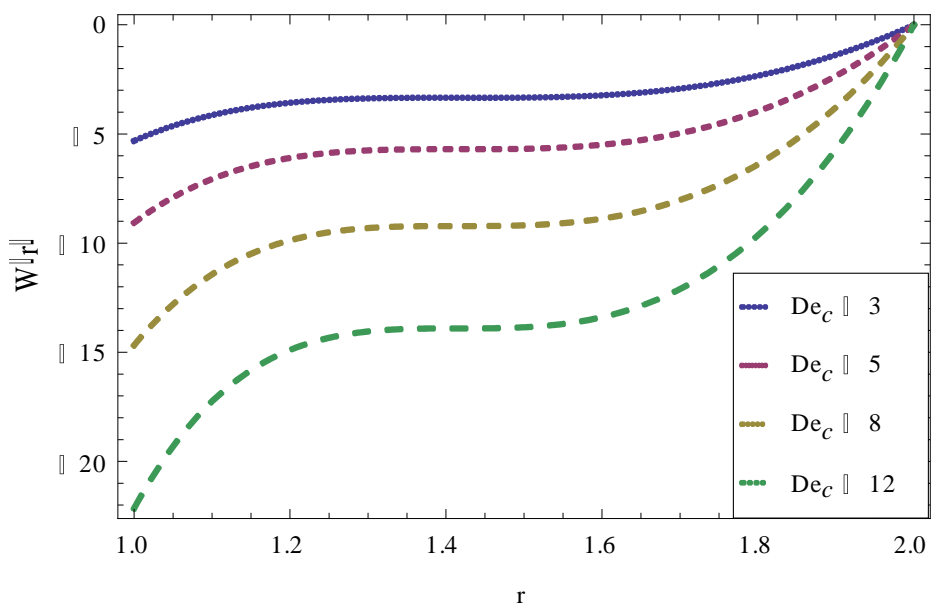

Figure 3: Dimensionless velocity profiles for different values of $D e_{c}=3,5,8,12$, at fixed values of $\delta=2, X=0.5, \eta_{0}=0.7$. 


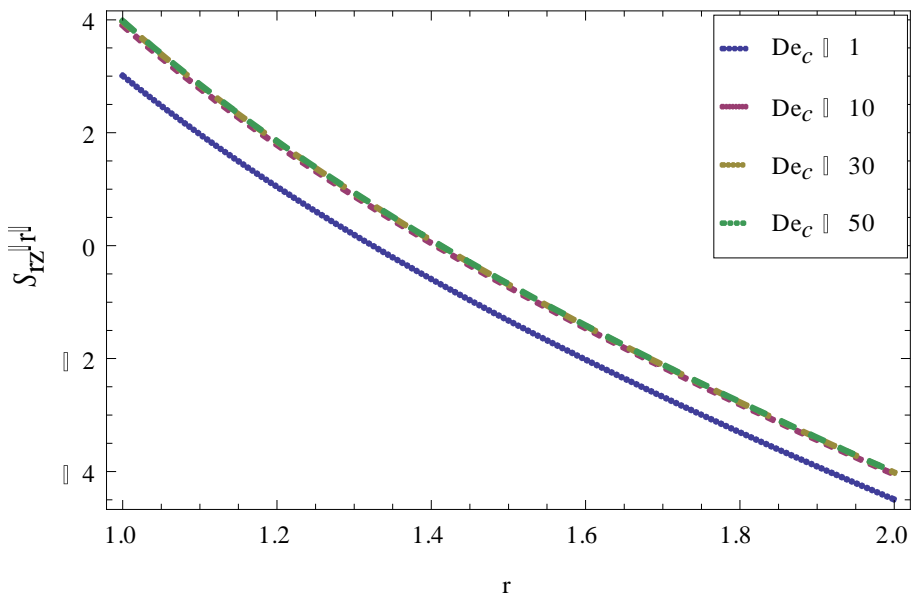

Figure 4: Dimensionless shear stress profiles for different values of $D e_{c}=1,10,30,50$, at fixed values of $\delta=2, X=0.5, \eta_{0}=0.7$.

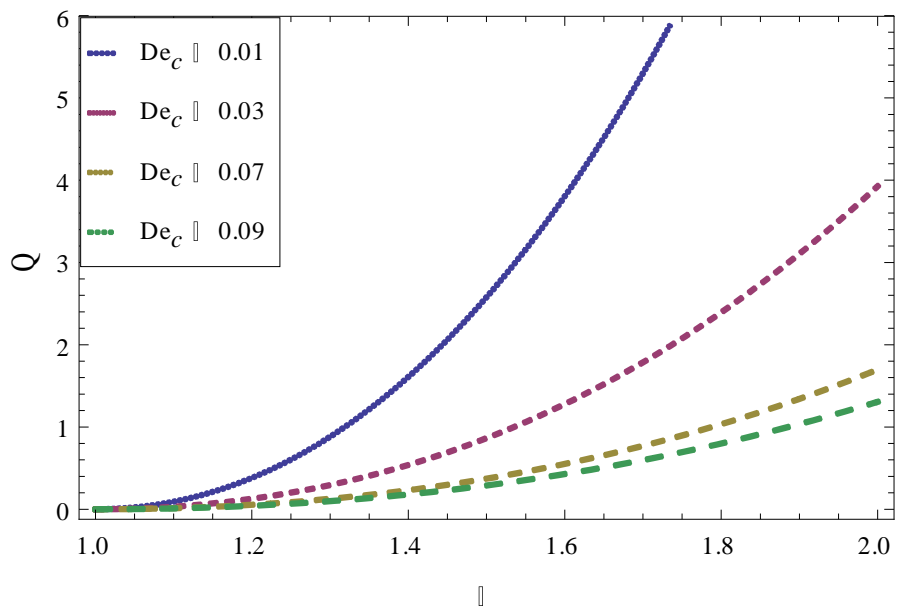

Figure 5: Dimensionless volume flow rates versus ratio of the radii for different values of $D e_{c}=0.01,0.03,0.07,0.09$, at fixed values of $X=0.5, \eta_{0}=0.7$. 


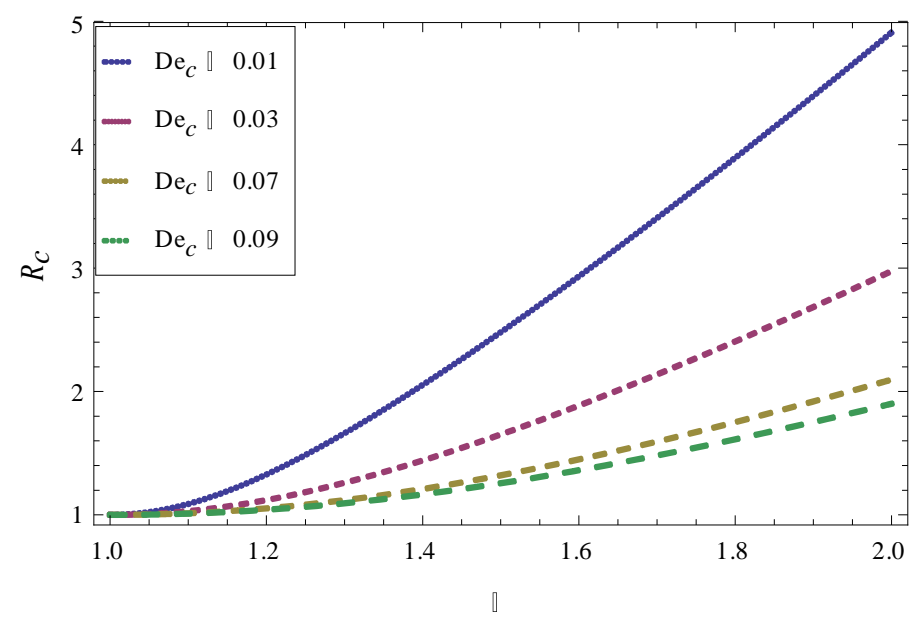

Figure 6: Thickness of the coated wire versus ratio of the radii for different values of $D e_{c}=0.01,0.03,0.07,0.09 x$ at fixed values of $X=0.5, \eta_{0}=0.7$.

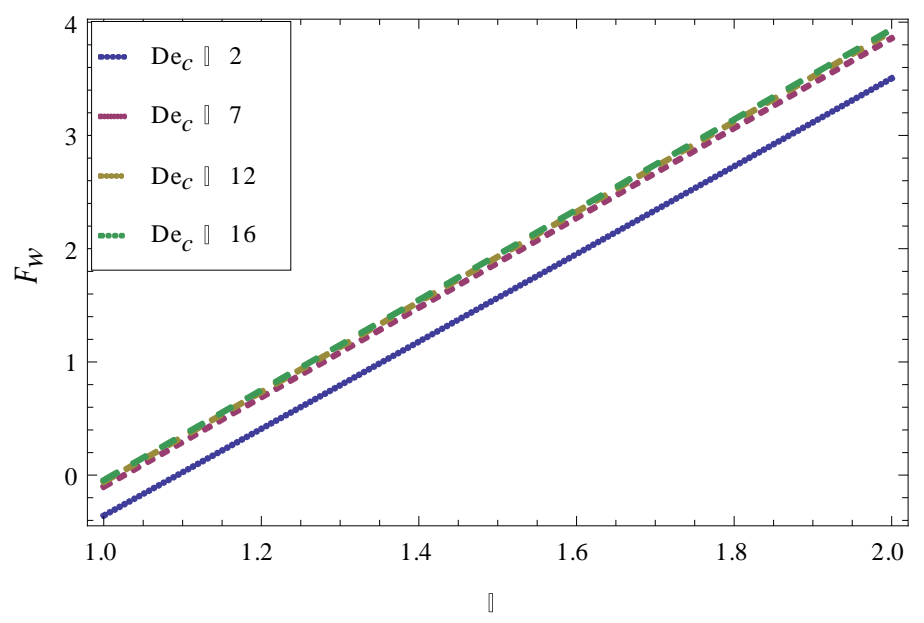

Figure 7: Force on the surface of the total wire for different values of $D e_{c}=2,7,12,16$, at fixed values of $X=0.5, \eta_{0}=0.7$. 


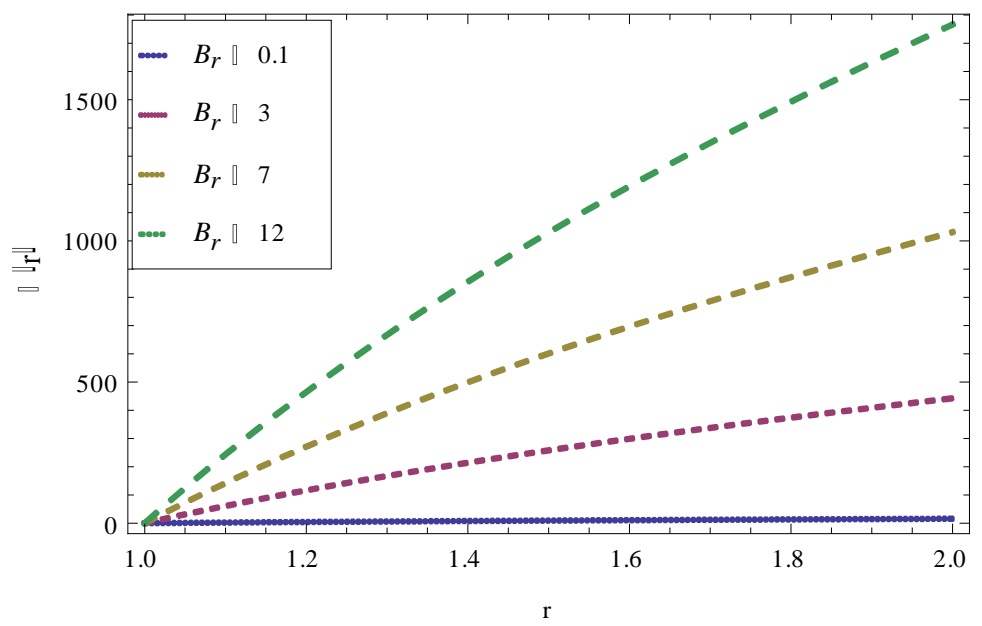

Figure 8: Dimensionless temperature distribution for different values of Brinkman number $B r=0.1,3,7,12$, at fixed values of $X=0.5, \delta=2, \eta_{0}=0.7, D e_{c}=3$.

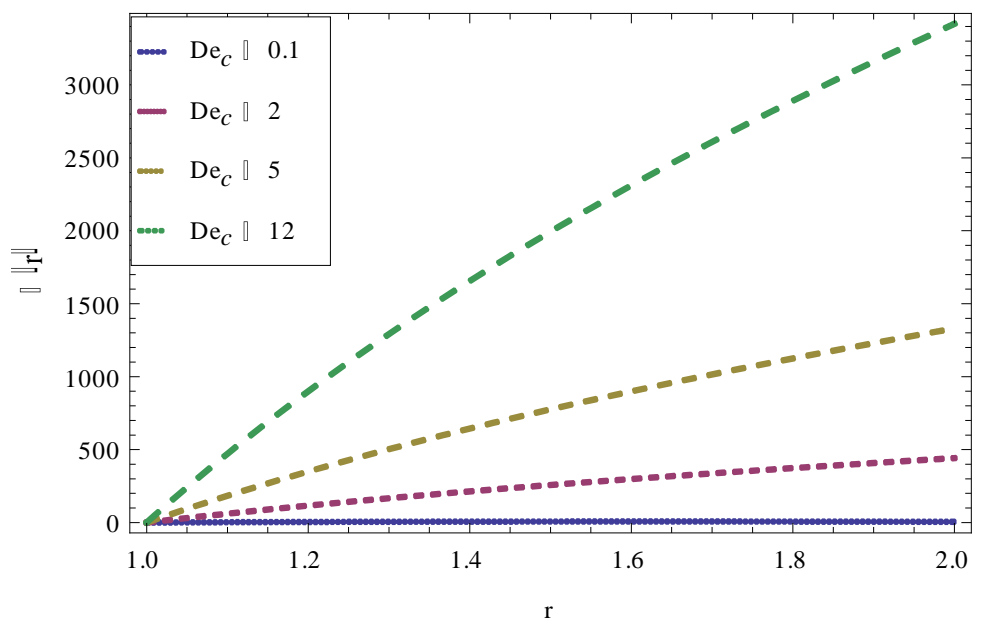

Figure 9: Dimensionless temperature distribution for different values of $D e_{c}=0.1,2,5,12$, at fixed values of $\delta=2, X=0.5, \eta_{0}=0.7, B r=5$. 


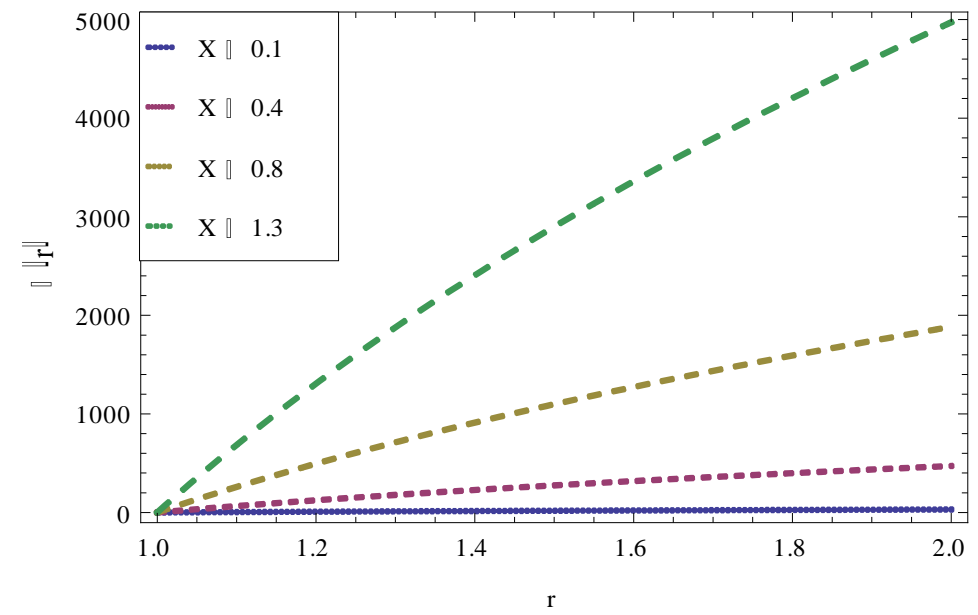

Figure 10: Dimensionless temperature distribution for different values of $X=0.1,0.4,0.8,1.3$, at fixed values of $\delta=2, D e_{c}=3, \eta_{0}=0.7, B r=5$.

5. Conclusion. Analytical solutions are derived for the flow of nonlinear Ellis fluids model in wire coating analysis by taking a special case for $\alpha=2$, after the application of Binomial series technique. Expressions are presented for the radial variation of the axial velocity and the temperature distribution. In engineering point of view, some results are also derived like volume flow rate, average velocity, shear stress, thickness of coated wire and force on the total wire. It was found that the axial velocity depends upon the dimensionless parameters $X$ and $D e_{c}$. Moreover, the velocity profile increases with different (increasing) values of the parameter $X$ and decreases with $D e_{c}$. It is to be noted that the shear stress, volume flow rate and thickness of the coated wire decreases for increasing values of $D e_{c}$. The dimensionless force on the total wire shows increase as $D e_{c}$ increases. Finally, the fluid temperature depends upon the dimensionless numbers $B r_{v} X$, $D e_{c}$ and it increases with increasing values of these parameters.

\section{REFERENCES}

[1]. Denn, M. M. (1980). Process fluid mechanics. Prentice Hall.

[2]. Middleman, S., Greener, J., \& Malone, M. (1977). Fundamentals of polymer processing (p. 468). New York: McGraw-Hill.

[3]. Akter, S., \& Hashmi, M. S. J. (1999). Analysis of polymer flow in a conical coating unit: a power law approach. Progress in organic coatings, 37(1), 15-22.

[4]. Akter, S., \& Hashmi, M. S. J. (1997). Plasto-hydrodynamic pressure distribution in a tepered geometry wire coating unit. In Proceedings of the 14th Conference of the Irish manufacturing committee (IMC14), Dublin (pp. 331-340).

[5]. Fenner, R. T., \& Williams, J. G. (1967). Analytical methods of wire-coating die design. Transactions And Journal of The Plastics Institute, 35(119), 701.

[6]. Siddiqui, A. M., Haroon, T., \& Khan, H. (2009). Wire coating extrusion in a pressure-type die in flow of a third grade fluid via homotopy perturbation method. International Journal of Nonlinear Sciences and Numerical Simulation, 10(2), 247-258.

[7]. Sajid, M., Siddiqui, A. M., \& Hayat, T. (2007). Wire coating analysis using MHD Oldroyd 8-constant fluid. International Journal of Engineering Science,45(2), 381-392. 
[8]. Katta, P., Alessandro, M., Ramsier, R. D., \& Chase, G. G. (2004). Continuous electrospinning of aligned polymer nanofibers onto a wire drum collector. Nano letters, 4(11), 2215-2218.

[9]. Kasajima, M. A. S. A. Y. U. K. I., \& Ito, K. A. T. S. U. H. I. K. O. (1973, October). Posttreatment of polymer extrudate in wire coating. In Appl Poly Symp (Vol. 20, pp. 221-235).

[10]. Bigg, D. M. (1977). Rheology and wire coating of high atomic number metal-low density polyethylene composites. Polymer Engineering \& Science, 17(10), 745-750.

[11]. Caswell, B., \& Tanner, R. I. (1978). Wirecoating die design using finite element methods. Polymer Engineering \& Science, 18(5), 416-421.

[12]. Roy, S. C., \& Dutt, D. K. (1981). Wire coating by withdrawal from a bath of power law fluid. Chemical Engineering Science, 36(12), 1933-1939.

[13]. Mitsoulis, E. (1986). Fluid flow and heat transfer in wire coating: a review.Advances in Polymer Technology, 6(4), 467-487.

[14]. Shah, R. A., Islam, S., Siddiqui, A. M., \& Haroon, T. (2011). Optimal homotopy asymptotic method solution of unsteady second grade fluid in wire coating analysis. Journal of the Korean Society for Industrial and Applied Mathematics, 15(3), 201-222.

[15]. Shah, R. A., Islam, S., Siddiqui, A. M., \& Haroon, T. (2012). Wire coating analysis with Oldroyd 8-constant fluid by Optimal Homotopy Asymptotic Method. Computers \& Mathematics with Applications, 63(3), 695-707.

[16]. Shah, R. A., Islam, S., \& Siddiqui, A. M. (2013). Exact solution of a differential equation arising in the wire coating analysis of an unsteady second grade fluid. Mathematical and Computer Modelling, 57(5), 1284-1288.

[17]. MacKelvey, J. M. (1962). Polymer processing. Wiley.

[18]. Bernhardt, E. C. (Ed.). (1959). Processing of thermoplastic materials (pp. 500-501). New York: Reinhold.

[19]. Bird, R. B. (2002). Transport phenomena. Applied Mechanics Reviews,55(1), R1-R4.

[20]. Rajagopal, K. R. (1993). Mechanics of non-Newtonian fluids. Pitman Research Notes in Mathematics Series, 129-129.

[21]. Bird, R. B., Armstrong, R. C., Hassager, O., \& Curtiss, C. F. (1977).Dynamics of polymeric liquids (Vol. 1, p. 210). New York: Wiley. 